\title{
MHD INSTABILITIES IN TOKAMAKS
}

\author{
H.J. de Blank \\ FOM Institute DIFFER - Dutch Institute for Fundamental Energy Research, \\ Association EURATOM-FOM, P.O. Box 1207, 3430 BE Nieuwegein, The Netherlands, www. differ.nl.
}

\begin{abstract}
A general introduction to ideal magnetohydrodynamic (MHD) stability of tokamak plasmas is given, using linear perturbations of the ideal MHD equations. Subsequently the Energy Principle for ideal MHD instabilities is derived. The specific instabilities which are then discussed are loosely divided into two categories. Under the name "current driven instabilities", external and internal kink modes, which are modes with a large radial extent, are discussed. The internal $m=1$ kink mode is responsible for sawtooth collapses and fishbone oscillations in tokamaks. Under the header "pressure driven instabilities", more localized modes are presented. These modes may limit the pressure gradient in the plasma without causing sizeable disruptions. The ballooning limit and the Mercier criterion are presented. The Troyon limit is mentioned as a synthesis of several of these stability boundaries.
\end{abstract}

\section{GENERAL THEORY OF MHD INSTABILITIES}

\section{A. The stability problem}

In magnetically confined plasmas, the optimization of the plasma density and temperature for fusion energy production has lead to a wide range of plasma instabilities. The adaptation of current and pressure profiles to avoid one type of instability can lead to yet another type of instability. The fastest instabilities in magnetically confined plasmas are usually MHD instabilities, and part of this lecture describes how to avoid them.

The main question in MHD stability theory is to consider an MHD equilibrium (measured or computed), and to predict if it is stable or unstable. The obvious approach is to simulate the evolution of the plasma numerically. The simulation may show growing instabilities and their longterm fate: saturation, triggering of other instabilities, or turbulence. However, this essentially nonlinear modelling is computationally expensive, especially when a wide range of length or time scales are involved.

The present lecture focusses on linear instabilities instead. This approach is systematic and decides if infinitesimal perturbations of an equilibrium are stable (wave-like, oscillating) or unstable (exponentially growing).

However, many other (usually slower) instabilities have been discovered that depend on physics ingredients that are not part of ideal MHD: electric resistivity, drift waves, and energetic (not thermalized) particles [1]. Yet, these instabilities often look very much like MHD instabilities if one considers the plasma motion, electric currents, and magnetic field perturbations. The second purpose of this lecture is therefore, to learn about the structure of MHD instabilities. In experiments, this helps to understand how a variety of instabilities show up in diagnostic signals.

\section{B. Ideal MHD}

Starting point is the set of equations of resistive MHD:

$$
\begin{aligned}
\frac{\partial \rho}{\partial t} & =-\nabla \cdot(\rho \boldsymbol{U}), \\
\frac{\partial p}{\partial t} & =-\boldsymbol{U} \cdot \nabla p-\gamma p \nabla \cdot \boldsymbol{U}, \quad \gamma=5 / 3, \\
\rho\left(\frac{\partial \boldsymbol{U}}{\partial t}+\boldsymbol{U} \cdot \nabla \boldsymbol{U}\right) & =\boldsymbol{j} \times \boldsymbol{B}-\nabla p, \\
\frac{\partial \boldsymbol{B}}{\partial t} & =-\nabla \times \boldsymbol{E}, \\
\boldsymbol{E} & =\eta \boldsymbol{j}-\boldsymbol{U} \times \boldsymbol{B}, \\
\boldsymbol{j} & =\nabla \times \boldsymbol{B} .
\end{aligned}
$$

In addition there is Gauss' law $\nabla \cdot \boldsymbol{B}=0$ which, once satisfied, is conserved by Faraday's law. In hot plasmas, the electric resistivity $\eta$ is negligible for sufficiently fast plasma processes. Taking $\eta=0$ in Ohm's law, we obtain the ideal MHD model. Introducing the total time derivative $d / d t \equiv \partial / \partial t+\boldsymbol{U} \cdot \nabla$, the ideal MHD equations can be written as

$$
\begin{aligned}
\rho \frac{d \boldsymbol{U}}{d t} & =\boldsymbol{B} \cdot \nabla \boldsymbol{B}-\nabla\left(s \rho^{\gamma}+\frac{1}{2} B^{2}\right), \\
\frac{d \rho}{d t} & =-\rho \nabla \cdot \boldsymbol{U}, \\
\frac{d s}{d t} & =0, \quad s \equiv \frac{p}{\rho^{\gamma}}, \\
\frac{d \boldsymbol{B}}{d t} & =\boldsymbol{B} \cdot \nabla \boldsymbol{U}-\boldsymbol{B} \nabla \cdot \boldsymbol{U} .
\end{aligned}
$$

where $s$ is the entropy density of the plasma. The momentum balance equation (1) is central to the MHD physics: it gives the evolution of the plasma flow $\boldsymbol{U}$ in terms of the density $\rho$, the magnetic field $\boldsymbol{B}$, and the entropy $s$ (or pressure $p=s \rho^{\gamma}$ ).

An important property of the MHD model is that the other three equations (the mass continuity equation (2), the 
energy equation of state (3), and Faraday's law (4)), give the evolution of $\rho, s$ (or $p$ ), and $\boldsymbol{B}$ due to the plasma flow as local conservation laws.

This is most easily seen for the energy equation (3), which merely states that the entropy density $s$ is conserved in each point as it moves along with the plasma velocity $\boldsymbol{U}$. For the other conservation laws, we first specify how an infinitesimal line element $d \boldsymbol{x}$ moves with the plasma flow $\boldsymbol{U}$,

$$
\begin{aligned}
\frac{d}{d t} d \boldsymbol{x} & =\frac{d}{d t}(\boldsymbol{x}+d \boldsymbol{x})-\frac{d \boldsymbol{x}}{d t} \\
& =\boldsymbol{U}(\boldsymbol{x}+d \boldsymbol{x})-\boldsymbol{U}(\boldsymbol{x}) \\
& =d \boldsymbol{x} \cdot \nabla \boldsymbol{U} .
\end{aligned}
$$

By constructing infinitesimal surface and volume elements out of line elements, $d^{2} \boldsymbol{x}=d \boldsymbol{x}_{1} \times d \boldsymbol{x}_{2}$ and $d^{3} x=d \boldsymbol{x}_{1} \times$ $d \boldsymbol{x}_{2} \cdot d \boldsymbol{x}_{3}$, we obtain

$$
\begin{aligned}
\frac{d}{d t} d^{2} \boldsymbol{x} & =-\left(d^{2} \boldsymbol{x} \times \nabla\right) \times \boldsymbol{U} \\
& =d^{2} \boldsymbol{x} \nabla \cdot \boldsymbol{U}-(\nabla \boldsymbol{U}) \cdot d^{2} \boldsymbol{x}, \\
\frac{d}{d t} d^{3} x & =d^{3} x \nabla \cdot \boldsymbol{U} .
\end{aligned}
$$

Combining expression (6) with (2), one finds the mass conservation law in integral form,

$$
\frac{d}{d t} \int \rho d^{3} x=0
$$

for any volume that moves with the plasma flow. In the same way we can combine Eqs. (4) and (5) to give

$$
\frac{d}{d t} \int \boldsymbol{B} d^{2} \boldsymbol{x}=0
$$

This equation states that the magnetic flux through an arbitrary surface that moves with the plasma is conserved.

To understand the consequences of this flux conservation law, consider the surface of a thin tube that surrounds a stretch of magnetic field line. By construction, there is zero magnetic flux crossing the surface. In addition, Gauss' law states that the flux that enters one end of the tube equals the flux that leaves the other end. Let this tube flow with the plasma velocity as time proceeds. The flux conservation law dictates that the flux that crosses the tube will remain zero and that the flux through the tube will remain the same. Hence, also at later times, the moving tube will precisely surround a magnetic field line. We can therefore say that magnetic field lines move with the plasma flow in ideal MHD. It follows that in an ideal MHD plasma, magnetic field lines cannot be created or annihilates, nor can they break up and reconnect. The magnetic topology is conserved, "frozen in the fluid", so to speak.

Strictly speaking, ideal MHD instabilities cannot change the magnetic topology of nested toroidal surfaces in a tokamak plasma. Thus, in a very hot (e.g. fusion-) plasma with negligible resistivity, ideal MHD instabilities may not seem to be dangerous to plasma confinement. However, as we shall see later, MHD instabilities may involve "magnetic resonant surfaces" in the plasma, where the plasma motion induces a narrow layer with very high current density. In such a layer, even very low resistivity may be sufficient to cause magnetic reconnection. Although this reconnection of field lines is confined to a thin layer, these field lines may extend into regions where they are far apart. Therefore, localized reconnection may have global consequences for particle and energy confinement.

The topological constraints can prevent ideal MHD instabilities altogether, even if there is plenty of (magnetic and kinetic) free energy to drive instabilities, as is often the case in magnetically confined (fusion-) plasmas. In such cases there may be a much slower, resistive MHD-instability, for which magnetic reconnection (again, taking place in a thin resonant layer) is essential. One such instability is the tearing mode, presented in another lecture [2].

Another important property of the ideal MHD system is that it can be derived from Hamilton's principle: the plasma motion $\boldsymbol{U}(\boldsymbol{x}, t)$ that makes the action

$$
S=\int_{t_{0}}^{t_{1}} L d t
$$

stationary, where the Lagrangian is $[3,4]$

$$
L=\int d^{3} x\left(\frac{1}{2} \rho U^{2}-\frac{p}{\gamma-1}-\frac{1}{2} B^{2}\right),
$$

is the true dynamical motion that satisfies the MHD equations. Here, it is understood that the plasma motion determines the evolution of $\rho, p$, and $\boldsymbol{B}$ through Eqs. (2), (3), and (4), respectively.

In order to demonstrate that Hamilton's principle for the Lagrangian (9) indeed produces the MHD momentum equation (1), we investigate how the action $S$ changes if the MHD fields are perturbed. Since all MHD quantities respond to the plasma motion, the primary perturbation is an arbitrary infinitesimal displacement $\boldsymbol{\xi}(\boldsymbol{x}, t)$ of the plasma fluid. We introduce the operator $\delta_{\xi} X$, the Lagrangian perturbation of a variable $X$, which is the change in the quantity while following the perturbed plasma motion. For instance, $\delta_{\xi} \boldsymbol{x}=\boldsymbol{\xi}$. It is helpful to introduce also the Eulerian perturbation $\delta_{\xi}^{E} \equiv \delta_{\xi}-\boldsymbol{\xi} \cdot \nabla$, which gives the perturbation at a fixed point in space, $\delta_{\xi}^{E} \boldsymbol{x}=0$. It therefore commutates with partial space and time derivatives,

$$
\delta_{\xi}^{E} \nabla=\nabla \delta_{\xi}^{E}, \quad \delta_{\xi}^{E} \frac{\partial}{\partial t}=\frac{\partial}{\partial t} \delta_{\xi}^{E} .
$$

While the Lagrangian perturbation does not commutate with $\nabla$ and $\partial / \partial t$, it commutates with the total derivative instead,

$$
\delta_{\xi} \frac{d}{d t}=\frac{d}{d t} \delta_{\xi}, \quad \text { since } \quad \delta_{\xi} \boldsymbol{U}=\frac{d \boldsymbol{\xi}}{d t} .
$$

An infinitesimal line element varies as $\delta_{\xi} d \boldsymbol{x}=d \boldsymbol{x} \cdot \nabla \boldsymbol{\xi}$. With these tools, one can obtain the perturbed density, pressure, 
and magnetic field from Eqs. (7), (3), and (8) respectively,

$$
\begin{aligned}
\delta_{\xi} \rho & =-\rho \nabla \cdot \boldsymbol{\xi}, \\
\delta_{\xi} p & =-\gamma p \nabla \cdot \boldsymbol{\xi}, \quad\left(\delta_{\xi} s=0\right) \\
\delta_{\xi} \boldsymbol{B} & =\boldsymbol{B} \cdot \nabla \boldsymbol{\xi}-\boldsymbol{B} \nabla \cdot \boldsymbol{\xi} .
\end{aligned}
$$

The perturbed volume element is given by $\delta_{\xi} d^{3} x=d^{3} x \nabla \cdot \boldsymbol{\xi}$. Now we can obtain the perturbed Lagrangian from Eq. (9),

$$
\begin{aligned}
\delta_{\xi} L= & \int d^{3} x\left[(\nabla \cdot \boldsymbol{\xi})\left(\frac{1}{2} \rho U^{2}-\frac{p}{\gamma-1}-\frac{1}{2} B^{2}\right)\right. \\
& \left.+\delta_{\xi}\left(\frac{1}{2} \rho U^{2}-\frac{p}{\gamma-1}-\frac{1}{2} B^{2}\right)\right] \\
= & \int d^{3} x\left[\rho \boldsymbol{U} \cdot \frac{d \boldsymbol{\xi}}{d t}+\left(p+\frac{1}{2} B^{2}\right) \nabla \cdot \boldsymbol{\xi}-\boldsymbol{B} \cdot(\boldsymbol{B} \cdot \nabla \boldsymbol{\xi})\right] \\
= & \int d^{2} \boldsymbol{x} \cdot \boldsymbol{\xi}\left(p+\frac{1}{2} B^{2}\right)+\frac{d}{d t} \int d^{3} x \rho \boldsymbol{U} \cdot \boldsymbol{\xi} \\
& -\int d^{3} x \boldsymbol{\xi} \cdot\left(\rho \frac{d \boldsymbol{U}}{d t}-\boldsymbol{j} \times \boldsymbol{B}+\nabla p\right) .
\end{aligned}
$$

In the last step we have used the mass conservation equation (2). The first term in (13) vanishes upon integration if one considers only internal perturbations, i.e., $\boldsymbol{\xi} \cdot \boldsymbol{n}=0$ on the plasma boundary (also $\rho=0$ and $\boldsymbol{B} \cdot \boldsymbol{n}=0$ there). Hamilton's principle can be extended to free-boundary perturbations (with $\boldsymbol{\xi} \cdot \boldsymbol{n} \neq 0$ ) by adding to the Lagrangian the vacuum magnetic energy that surrounds the plasma. The second term in (13) does not contribute to the perturbed action $\delta_{\xi} S=\int \delta_{\xi} L d t$ if the perturbation is zero at $t=t_{0}$ and $t=t_{1}$. The term vanishes altogether if one considers perturbations of a static equilibrium. The third term vanishes for arbitrary $\boldsymbol{\xi}$ if and only if the momentum balance equation (1) is satisfied. In this way Eq. (1) follows from Hamilton's principle.

\section{The linearized MHD equations}

We now posess the tools to study MHD waves and instabilities. Perturbing the momentum equation (1) gives us the equation of motion for $\boldsymbol{\xi}$,

$$
\begin{aligned}
0 & =\delta_{\xi}\left(\rho \frac{d \boldsymbol{U}}{d t}-\boldsymbol{j} \times \boldsymbol{B}+\nabla p\right) \\
& =\rho \frac{d^{2} \boldsymbol{\xi}}{d t^{2}}-\boldsymbol{F}(\boldsymbol{\xi})
\end{aligned}
$$

where the linear force operator $\boldsymbol{F}$ is defined as

$$
\begin{aligned}
\boldsymbol{F}(\boldsymbol{\xi})= & \delta_{\xi}(\boldsymbol{j} \times \boldsymbol{B}-\nabla p)+\rho \frac{d \boldsymbol{U}}{d t} \nabla \cdot \boldsymbol{\xi} \\
= & (\nabla \times \boldsymbol{Q}) \times \boldsymbol{B}+(\nabla \times \boldsymbol{B}) \times \boldsymbol{Q}+ \\
& \nabla(\boldsymbol{\xi} \cdot \nabla p+\gamma p \nabla \cdot \boldsymbol{\xi})+\nabla \cdot\left(\rho \boldsymbol{\xi} \frac{d \boldsymbol{U}}{d t}\right),
\end{aligned}
$$

and where

$$
\boldsymbol{Q} \equiv \delta_{\xi}^{E} \boldsymbol{B}=\nabla \times(\boldsymbol{\xi} \times \boldsymbol{B})
$$

Since Eq. (14) is linear in $\boldsymbol{\xi}$, it determines eigenfunctions $\boldsymbol{\xi}(\boldsymbol{x}, t)=\boldsymbol{\xi}(\boldsymbol{x}) e^{-i \omega t}$

The force operator $\boldsymbol{F}$ possesses the important property that it is self-adjoint, i.e., given any two vector fields $\boldsymbol{\xi}$ and $\zeta$, the operator satisfies

$$
\int \boldsymbol{\zeta} \cdot \boldsymbol{F}(\boldsymbol{\xi}) d^{3} x=\int \boldsymbol{\xi} \cdot \boldsymbol{F}(\boldsymbol{\zeta}) d^{3} x
$$

Direct proofs of the self-adjointness of $\boldsymbol{F}$ can be found in many texts $[5,3,6,7,8,9]$. Most proofs require lengthy vector manipulations that seem to lack direction. We will therefore take a more instructive approach.

The key element of our proof is that the Lagrangian perturbations form a Lie algebra. Consider the commutator of two perturbations, $\left(\delta_{\zeta} \delta_{\xi}-\delta_{\xi} \delta_{\zeta}\right) \boldsymbol{x}=\delta_{\zeta} \boldsymbol{\xi}-\delta_{\xi} \boldsymbol{\zeta} \equiv \boldsymbol{\eta}$. This is equal to another perturbation of the position, $\boldsymbol{\eta}=\delta_{\eta} \boldsymbol{x}$. Since the perturbations of all MHD quantities derive from the displacement vector, we have the general operator identity

$$
\delta_{\zeta} \delta_{\xi}-\delta_{\xi} \delta_{\zeta}=\delta_{\eta}, \quad \boldsymbol{\eta} \equiv \delta_{\zeta} \boldsymbol{\xi}-\delta_{\xi} \boldsymbol{\zeta}
$$

which defines the Lie algebra. Now consider the double variation of the Lagrangian (13) and use the boundary conditions,

$$
\begin{aligned}
\delta_{\zeta} \delta_{\xi} L & =\delta_{\zeta} \int d^{3} x\left[\frac{\partial}{\partial t}(\rho \boldsymbol{U} \cdot \boldsymbol{\xi})-\boldsymbol{\xi} \cdot\left(\rho \frac{d \boldsymbol{U}}{d t}-\boldsymbol{j} \times \boldsymbol{B}+\nabla p\right)\right] \\
& =\int d^{3} x\left[\frac{\partial}{\partial t}\left(\rho \frac{d \boldsymbol{\zeta}}{d t} \cdot \boldsymbol{\xi}\right)-\boldsymbol{\xi} \cdot\left(\rho \frac{d^{2} \boldsymbol{\zeta}}{d t^{2}}-\boldsymbol{F}(\boldsymbol{\zeta})\right)\right] \\
& =2 K(\boldsymbol{\zeta}, \boldsymbol{\xi})-2 \delta W(\boldsymbol{\zeta}, \boldsymbol{\xi}),
\end{aligned}
$$

where we have defined the kinetic and potential energy functionals [5],

$$
\begin{aligned}
K(\boldsymbol{\zeta}, \boldsymbol{\xi}) & =\frac{1}{2} \int \rho \frac{d \boldsymbol{\zeta}}{d t} \cdot \frac{d \boldsymbol{\xi}}{d t} d^{3} x, \\
\delta W(\boldsymbol{\zeta}, \boldsymbol{\xi}) & =-\frac{1}{2} \int \boldsymbol{\zeta} \cdot \boldsymbol{F}(\boldsymbol{\xi}) d^{3} x .
\end{aligned}
$$

According to the Lie algebra, $\delta_{\zeta} \delta_{\xi} L-\delta_{\xi} \delta_{\zeta} L=\delta_{\eta} L$. Moreover, $\delta_{\eta} L=0$ because the unperturbed plasma satisfies the momentum equation (1). Therefore, $\delta_{\zeta} \delta_{\xi} L=\delta_{\xi} \delta_{\zeta} L$, and since the kinetic energy functional $K$ is manifestly selfadjoint, it follows that the potential energy functional $\delta W$ is symmetric,

$$
\delta W(\boldsymbol{\zeta}, \boldsymbol{\xi})=\delta W(\boldsymbol{\xi}, \boldsymbol{\zeta}) .
$$

This concludes the proof that the force operator (15) is selfadjoint.

\section{The Energy Principle}

The self-adjointness (16) of the force operator has several important consequences that are useful in the stability analysis of actual configurations. Here, we list four properties. 
1. For a normal mode $\boldsymbol{\xi}(\boldsymbol{x}, t)=\boldsymbol{\xi}(\boldsymbol{x}) e^{-i \omega t}, \omega^{2}$ is always real. It easy to see that $\omega^{* 2}=\omega^{2}$ from

$$
\begin{aligned}
& \omega^{2} \int \rho \boldsymbol{\xi}^{*} \cdot \boldsymbol{\xi} d^{3} x=-\int \boldsymbol{\xi}^{*} \cdot \boldsymbol{F}(\boldsymbol{\xi}) d^{3} x \\
& =-\int \boldsymbol{\xi} \cdot \boldsymbol{F}\left(\boldsymbol{\xi}^{*}\right) d^{3} x=\omega^{* 2} \int \rho \boldsymbol{\xi}^{*} \cdot \boldsymbol{\xi} d^{3} x .
\end{aligned}
$$

The first step uses the eigenvalue equation for the mode, $-\rho \omega^{2} \boldsymbol{\xi}=\boldsymbol{F}(\boldsymbol{\xi})$, the second step self-adjointness, and the third step the complex conjugate of (14). Because of $\mathbf{1}$. an eigenmode is either oscillating ( $\omega$ real) or exponentially growing ( $\omega$ imaginary). Overstable modes (growing oscillations) cannot occur. Hence if a mode is stable it cannot become unstable by a slight variation of the equilibrium which would add a small imaginary component to a real frequency $\omega$. Instead, access to instability is only possible via marginal stability $(\omega=0)$, because $\omega^{2}$ has to change sign.

2. Eigenmodes with different eigenvalues are orthogonal,

$$
\int \rho \boldsymbol{\xi}_{m}^{*} \cdot \boldsymbol{\xi}_{n} d^{3} x=0, \quad \text { if } \quad \omega_{m}^{2} \neq \omega_{n}^{2} .
$$

3. The Energy Principle $[5,10]$ states that an equilibrium is stable if and only if

$$
\delta W\left(\boldsymbol{\xi}^{*}, \boldsymbol{\xi}\right) \geq 0
$$

for all possible displacements $\boldsymbol{\xi}$, which satisfy appropriate boundary conditions and are bounded in energy. We will prove first the sufficiency and then the necessity of the stability condition.

Sufficiency of the Energy Principle follows simply from conservation of the total energy $H=K(t)+\delta W(t)$. If $\delta W>0$ then $K(t)$ cannot grow beyond the initial total energy $H$, i.e., an exponentially growing instability is not possible. The necessity of the Energy Principle means that the equilibrium is unstable whenever we can find a trial function $\boldsymbol{\xi}_{T}$ (not necessarily an eigenmode) for which $\delta W<0$. In order to see this we consider the positive integral

$$
I(t) \equiv \frac{1}{2} \int \rho|\boldsymbol{\xi}|^{2} d^{3} x
$$

We now calculate $d^{2} I / d t^{2}$. Using Eqs. (14) and (1) to remove $d^{2} \boldsymbol{\xi} / d t^{2}$ terms we obtain

$$
\frac{d^{2} I}{d t^{2}}=2 K-2 \delta W
$$

Now take as initial condition $\partial \boldsymbol{\xi}_{T} / \partial t=0$, so that initially the kinetic energy $K$ is zero. Therefore the (conserved) total energy $H=K+\delta W$ is negative. Since at later times $K \geq 0$ we find

$$
\frac{d^{2} I}{d t^{2}}=4 K-2 H>-2 H>0,
$$

which proves that $I$ grows at least as fast as $t^{2}$ as $t \rightarrow \infty$. This proves the Energy Principle [11,7].
An advantage of the Energy Principle is that one can consider the sign of $\delta W$ for a class of cleverly chosen trial functions $\boldsymbol{\xi}_{T}$, which are however not eigenfunctions. (The eigenfunctions might be difficult to calculate). For such a limited class of trial functions the condition $\delta W>0$ is necessary for stability, but not sufficient. One obtains a stability boundary beyond which the equilibrium is definitely unstable. On the "stable" side of such aboundary, however, there might still be unstable modes that have been "overlooked" because they are not in the set of trial functions. An example of such a necessary but insufficient criterion is the Mercier criterion which we will encounter in section III..

4. In order to find the precise stability limits one has to minimize $\delta W$ for all allowed perturbations $\boldsymbol{\xi}$. This can be done in a standard way by variational calculus. The resulting Euler equation is precisely equation (14) at marginal stability,

$$
\boldsymbol{F}(\boldsymbol{\xi})=0 \text {. }
$$

In other words, finding stability boundaries by minimizing $\delta W$ and finding normal modes by solving Eq. (14) are equivalent. This fact will appear several times in the following sections.

\section{E. The intuitive form of the energy functional}

In this section and in the following one we consider the actual stability boundaries for tokamak configurations. Such stability limits are found by considering at marginal stability $(\omega=0)$ specific classes of modes that are expected to be the most unstable ones. Most of the following analysis will be done not on the basis of the marginal stability equation (18) but with the energy principle. Freidberg [6,7] will be followed here in discussing the intuitive form of the energy functional,

$$
\begin{aligned}
\delta W=\frac{1}{2} \int_{P} d^{3} x[ & \gamma p|\nabla \cdot \boldsymbol{\xi}|^{2}+\left|\boldsymbol{Q}_{\perp}\right|^{2}+B^{2}\left|\nabla \cdot \boldsymbol{\xi}_{\perp}+2 \boldsymbol{\xi}_{\perp} \cdot \boldsymbol{\kappa}\right|^{2} \\
& -j_{\|}\left(\boldsymbol{\xi}^{*} \times \boldsymbol{b} \cdot \boldsymbol{Q}_{\perp}\right) \\
& \left.-2\left(\boldsymbol{\xi}_{\perp} \cdot \nabla p\right)\left(\boldsymbol{\xi}_{\perp}^{*} \cdot \boldsymbol{\kappa}\right)\right]
\end{aligned}
$$

which can be obtained from expression (17) by putting $K=$ 0 and by performing several integrations by parts. Here, $\boldsymbol{b}=$ $\boldsymbol{B} / B$ is the unit vector parallel to the magnetic field. Its derivative along the field line $\boldsymbol{\kappa}=\boldsymbol{b} \cdot \nabla \boldsymbol{b}$ is the field line curvature. Note that $\boldsymbol{\kappa} \perp \boldsymbol{b}$. For the adjoint perturbation we have taken the complex conjugate, $\xi^{A}=\boldsymbol{\xi}^{*}$. The Eulerian perturbation of the magnetic field $Q$ is given after Eq. (15).

The first three terms in the integrand of (19) are always positive and stabilizing. The last two terms are potentially destabilizing.

The first stabilizing term represents plasma compression. It is an important stabilizing force in sound waves. At marginal stability, however, the kinetic energy being zero, it is the only term in which $\xi_{\|}$appears. All other terms depend on $\boldsymbol{\xi}_{\perp}$ only. One can minimize $\delta W$ once and for all with 
respect to $\xi_{\|}$. In a tokamak configuration $\xi_{\|}$can actually be chosen to make $\frac{1}{2} \int d^{3} x \gamma p|\nabla \cdot \boldsymbol{\xi}|^{2}=0$, by making $\nabla \cdot \boldsymbol{\xi}=0$ in the entire plasma with the possible exception of some isolated surfaces. It is a fortunate situation that we can consider the plasma as being incompressible because the ideal gas equation of state (3) has a rather limited range of validity in tokamaks in the low collisionality regime, especially in the presence of non-thermal particles due to non-Ohmic heating or fusion reactions. The second term is caused by the perturbed magnetic field component perpendicular to the equilibrium field. It is the energy required to bend magnetic field lines. It is the dominant stabilizing term in shear Alfvén waves. The third term involves the parallel component of the perturbed magnetic field and therefore the perturbation of the magnetic field strength $B$. Since $B$ can be viewed as the density of field lines, this term is caused by the compression of field lines, countered by the magnetic pressure. This term dominates compressional Alfvén waves.

The fourth term can be destabilizing. Because it is proportional to $j_{\|}$, modes driven unstable by it are called current driven instabilities. The fifth term is also a potential source of instabilities, called pressure driven modes because the term is proportional to the pressure gradient. We will consider both classes of instabilities in more detail.

\section{CURRENT DRIVEN INSTABILITIES}

\section{A. Introduction}

In this section we will consider instabilities which are driven by the energy stored in the current parallel to the magnetic field, i.e., by the fourth term in the energy functional (19). The driving force is due to the tendency of two conducting wires (read: flux tubes) with parallel currents to repel each other. The destabilizing effect remains even if the plasma pressure is small. This justifies the distinction between current driven modes and pressure and driven instabilities such as the ballooning modes.

We shall see that the current-driven modes tend to have very small parallel wave numbers, $\boldsymbol{b} \cdot \nabla \ll \nabla$, so that these modes must have almost the same helical structure as the magnetic field lines. In a large aspect ratio tokamak plasma $(a \ll R)$ with circular poloidal cross-section, the plasma locally has the approximate symmetry of a cylinder, and eigenmodes of the system can be approximated by a single poloidal harmonic $[12,13]$. Modes dominated by such a helical displacement $e^{i(m \theta-n \phi)}$ are called kink modes.

The next subsection gives a general approach to internal MHD instabilities with low poloidal and toroidal mode numbers $(m, n)$, i.e., with a wavelength comparable to the machine minor radius. Associated with the large spatial extent of the linear mode is usually also a large nonlinear amplitude of the instability. Therefore such instabilities can lead to a disruptive loss of confinement in a significant fraction of the plasma.
Most current-driven modes are stable in ideal MHD (i.e. without resisitivity), except free boundary modes, which are instabilities with a finite amplitude on the plasma surface and in the vacuum region. The vicinity of a conducting wall can be important in stabilizing these modes. They are discussed in the next subsection.

The final subsection discusses $m=1, n=1$ kink modes. These modes require a special treatment because they can also lead to internal instabilities, if a flux surface with $q=1$ is present in the plasma. The internal kink mode is one of the mechanisms that in tiokamaks drive internal disruptions or sawtooth collapses, so called because they occur in the hot plasma core repetatively, typically removing all the excess pressure from the center, leaving profiles with a flat or even hollow central part. The spatial extent of these modes is mainly determined by the plasma volume where the safety factor $q<1$. This volume usually contains a considerable fraction of the plasma kinetic energy. Sawtooth collapses often show fast initial growth of the perturbation. In the language of linear stability, a large growth rate means that the plasma is far in the unstable region in configuration space. Therefore, the mode is not triggered when the slowly evolving equilibrium crosses the stability boundary. Rather, some essentially nonlinear trigger mechanism has to take place, which is at present not understood. Hence, we have to keep in mind that linear stability theory does not give a description of the temporal behaviour of a disruptive instability. It can, however, give a good indication whether an instability can indeed occur, and in addition it can provide the spatial structure and growth rate of the mode at low amplitudes.

\section{B. Kink modes}

The most important features of current driven instabilities can be studied in the low- $\beta$ approximation, in which

$$
\boldsymbol{j}_{\perp}=\frac{\boldsymbol{B} \times \nabla p}{B^{2}}=\mathcal{O}\left(\varepsilon^{2}\right), \quad \varepsilon=\frac{a}{R_{0}} \ll 1 .
$$

An immediate consequence of $\beta=\mathcal{O}\left(\varepsilon^{2}\right)$ is that the Shafranov shift of the flux surfaces is small, $\Delta=\mathcal{O}\left(\varepsilon^{2} a\right)$. Therefore, if shaping effects such as elongation and triangularity are small, the flux surfaces have approximately centered circular cross sections [14]. In addition, since $\varepsilon$ is small, the field line curvature is small and $B(\sim 1 / R)$ does not vary much over the plasma cross section. Therefore, we can approximate the plasma by a cylindrical column of radius $r=a$ and length $2 \pi R_{0}$. Our cylindrical coordinates will be $(r, \theta, \phi)$, where $\theta$ is the poloidal angle and $\phi$ the longitudinal coordinate. Of course we impose periodic boundary conditions for $\phi \rightarrow \phi+2 \pi$. We will write vectors in column notation when they are given in terms of the orthonormal basis $\hat{\boldsymbol{r}}, \hat{\boldsymbol{\theta}}, \hat{\boldsymbol{\phi}}$. The magnetic field and the current density are

$\boldsymbol{B}=B\left(\begin{array}{c}0 \\ \frac{r}{q R_{0}} \\ 1\end{array}\right)+\mathcal{O}\left(\varepsilon^{2}\right), \quad \boldsymbol{j}=\frac{B}{R}\left(\begin{array}{c}0 \\ 0 \\ \frac{1}{r}\left(\frac{r^{2}}{q}\right)^{\prime}\end{array}\right)+\mathcal{O}\left(\varepsilon^{2}\right)$. 
We will now look for the perturbations $\boldsymbol{\xi}$ that minimize $\delta W(19)$. We do this in four steps.

1. Due to the symmetry in both $\theta$ and $\phi$ we can consider single poloidal and toroidal harmonics, with mode numbers $(m, n)$ as normal modes,

$$
\boldsymbol{\xi}=\boldsymbol{\xi}(r) e^{i(m \theta-n \phi-\omega t)} .
$$

2. We write the vector $\boldsymbol{\xi}$ as

$$
\boldsymbol{\xi}=\xi_{r} \hat{\boldsymbol{r}}+\xi_{\theta} \hat{\boldsymbol{\theta}}+\xi_{\|} \boldsymbol{b} .
$$

The component $\xi_{\|}$occurs in $\delta W$ only in the plasma compression term $\int \gamma p|\nabla \cdot \boldsymbol{\xi}|^{2} d^{3} x$, which can be minimized to zero by choosing $\xi_{\|}$such that $\nabla \cdot \boldsymbol{\xi}=0$ in most of the volume. The remaining energy functional depends on $\xi_{r}$ and $\xi_{\theta}$ only.

3. As a consequence of the low- $\beta$ ordering, the stabilizing magnetic energy terms in $\delta W$ are by far the largest: all other terms are $\mathcal{O}\left(\varepsilon^{2}\right)$ smaller. Hence, minimization of $\delta W$ requires to leading order that $\frac{1}{2} \int|\boldsymbol{Q}|^{2} d^{3} x$ is minimized. We will see that this minimization poses only one condition on the two functions $\xi_{r}(r)$ and $\xi_{\theta}(r)$. After this first minimization we will consider the remaining terms in the energy, which are $\mathcal{O}\left(\varepsilon^{2}\right)$, and derive a stability criterion from them. The (Eulerian) perturbation of the magnetic field is

$$
\boldsymbol{Q}=\nabla \times(\boldsymbol{\xi} \times \boldsymbol{B})=\frac{B_{0}}{r}\left(\begin{array}{c}
-i\left(\frac{m}{q}-n\right) \frac{r}{R_{0}} \xi_{r} \\
\frac{r}{R_{0}}\left[\left(\frac{r}{q} \xi_{r}\right)^{\prime}+i n \xi_{\theta}\right] \\
-\left(r \xi_{r}\right)^{\prime}-i m \xi_{\theta}
\end{array}\right)
$$

One sees that the $\hat{\boldsymbol{\phi}}$ component is dominant. The $\hat{\boldsymbol{r}}$ and $\hat{\boldsymbol{\theta}}$ components are $\mathcal{O}(\varepsilon)$ smaller and give $\mathcal{O}\left(\varepsilon^{2}\right)$ smaller contributions to the energy. Hence, we arrive at the condition that the $\hat{\phi}$ component must be small, at most $\mathcal{O}(\varepsilon)$. At this point, we must be somewhat more careful. Our derivation so far is fine if we consider an unstable equilibrium and merely look for a trial function $\boldsymbol{\xi}_{T}$ that makes $\delta W\left(\boldsymbol{\xi}_{T}\right)<0$ in order to prove this. However, in order to obtain the actual stability criterion one needs to extend the expansion to higher order, $\mathcal{O}\left(\varepsilon^{2}\right)$, in the energy. Moreover, we want to find the actual eigenfunction $\boldsymbol{\xi}$. We want to know the spatial structure of the mode. In principle, we have to solve the original Euler equation $\boldsymbol{F}(\boldsymbol{\xi})=0$, all three components of it, in the three unknown components of $\boldsymbol{\xi}$. Here, another nice property of the energy principle comes to the rescue. After all, we already have arrived at a one-dimensional system with only two unknowns,

$$
\delta W\left(\xi_{r}, \xi_{\theta}\right)=2 \pi^{2} B_{0}^{2} \int \frac{R_{0}}{r}\left|\left(r \xi_{r}\right)^{\prime}+i m \xi_{\theta}\right|^{2} d r+\mathcal{O}\left(\varepsilon^{2}\right) .
$$

The usefulness of the energy approach is that we can readily extract Euler equations for $\xi_{r}$ and $\xi_{\theta}$ from this expression,

$$
\begin{aligned}
r \frac{d}{d r}\left[\frac{1}{r}\left(\left(r \xi_{r}\right)^{\prime}+i m \xi_{\theta}\right)\right] & =\mathcal{O}\left(\varepsilon^{2}\right) \\
-\frac{i m}{r}\left(\left(r \xi_{r}\right)^{\prime}+i m \xi_{\theta}\right) & =\mathcal{O}\left(\varepsilon^{2}\right) .
\end{aligned}
$$

To leading order, the two equations are not independent, and we will have to go to higher order in $\varepsilon$ to find an independent second equation. We discuss Eq. (20) for the cases $m=$ 0 and $m \neq 0$ separately. For $m=0$ we have $\left(r \xi_{r}\right)^{\prime}=$ 0 . The only solution that is regular in $r=0$ is $\xi_{r}=0$. This implies that $\boldsymbol{\xi}$ is always tangent to the flux surfaces and energy cannot be released: the $m=0$ modes are stable. For $m \neq 0$ we obtain

$$
\xi_{\theta}=\frac{i}{m}\left(r \xi_{r}\right)^{\prime}+\mathcal{O}\left(\varepsilon^{2}\right)
$$

Note that this equation does not merely reduce the dominant term, shown in Eq. (20), to the order of the other terms, $\mathcal{O}\left(\varepsilon^{2}\right)$. The term becomes much smaller than the rest of $\delta W, \mathcal{O}\left(\varepsilon^{4}\right)$. This is a general feature which occurs if one expands a bilinear energy functional in a small parameter. Another general pattern is that the orders of the energy terms are always even powers of the small parameters. It is also useful to note that the term that we have minimized is approximately the field line compression energy. Accordingly, Eq. (21) states that the plasma motion in the poloidal plane is to a good approximation incompressible, $\operatorname{Div}\left(\xi_{r}, \xi_{\theta}\right)=0$. 4. Using Eq. (21) we eliminate $\xi_{\theta}$ from $\delta W\left(\xi_{r}, \xi_{\theta}\right)$. This requires some integrations by parts and cancellation of $q^{\prime}$ terms. The result is

$$
\begin{aligned}
\delta W_{\mathrm{cyl}} & =\pi^{2} \frac{B_{0}^{2}}{R_{0}} \int_{0}^{a}\left(\left|r \xi_{r}^{\prime}\right|^{2}+\left(m^{2}-1\right)\left|\xi_{r}\right|^{2}\right)\left(\frac{n}{m}-\frac{1}{q}\right)^{2} r d r \\
& +\mathcal{O}\left(\varepsilon^{4}\right)
\end{aligned}
$$

Since $m \neq 0$, the dominant terms in expression (22) are clearly positive definite and therefore do not give rise to instabilities. We will now discuss two ways in which unstable modes can arise that are described by Eq. (22). The first possibility involves the boundary conditions. If the mode has a non-zero amplitude at the plasma boundary, $\xi_{r}(a) \neq 0$, then extra terms should be added to $\delta W_{\text {cyl }}$ corresponding to the energy of the vacuum region and of the plasma boundary itself. The additional terms can be negative and can give rise to external kink modes. These instabilities will be briefly discussed in the next subsection. The other possibility of instability is when $\delta W_{\text {cyl }}$ vanishes to leading order, which is only possible if $m=1$. Then the $\mathcal{O}\left(\varepsilon^{4}\right)$ terms become important, and these terms can be negative. This leads to the potentially unstable internal $m=1$ kink mode, which will be treated in the final subsection.

\section{External kink modes}

The computation of external kink modes can be performed using an extended energy principle, which takes into account the energy in the plasma, in the vacuum region, and in general a contribution from the plasma-vacuum boundary surface. The boundary conditions between plasma and vacuum, and between vacuum and (conducting) wall, play a central role in the derivation of the extended energy functional. The 
first two of these conditions are that at both boundaries the magnetic field is tangent to the boundary surface. The third condition is that at the plasma-vacuum interface the momentum balance requires that the quantity $p+\frac{1}{2} B^{2}$ is continuous. We will give here the resulting energy functional for the case of a circular plasma cross section with minor radius $r=a$ and a perfectly conducting wall at $r=b$, which can be obtained following the steps outlined in the previous subsection [15].

$$
\begin{aligned}
& \delta W_{\text {external }}= \\
& \pi^{2} \frac{B_{0}^{2}}{R_{0}}\left\{\int_{0}^{a}\left(\left|r \xi_{r}^{\prime}\right|^{2}+\left(m^{2}-1\right)\left|\xi_{r}\right|^{2}\right)\left(\frac{n}{m}-\frac{1}{q}\right)^{2} r d r+\right. \\
&\left.\left.\quad\left[\frac{2}{q}\left(\frac{n}{m}-\frac{1}{q}\right)+(1+m \lambda)\left(\frac{n}{m}-\frac{1}{q}\right)^{2}\right] r^{2}\left|\xi_{r}\right|^{2}\right|_{r=a}\right\},
\end{aligned}
$$

where

$$
\lambda=\frac{1+(a / b)^{2 m}}{1-(a / b)^{2 m}} .
$$

The contribution of $\lambda>0$ is the stabilizing effect of the wall. The first term in the second line of Eq. (C.) is destabilizing if $q(a)<m / n$. All other terms are positive. For fixed $m / n$, the mode with the lowest poloidal mode numbers has the lowest field line bending energy and is therefore the least stable one. Potentially the most unstable external kink modes are the $m=2, n=1$ mode and in particular the $m=1, n=1$ mode. They are also the most dangerous modes since they affect a large part of the plasma and can cause a violent disruption that terminates the discharge and can damage tokamak components. These modes require $q(a)<2$ and $q(a)<1$, respectively. As soon as $q(a)$ drops below 2 , the $m=2$ mode becomes unstable unless the current profile is extremely peaked or a conducting shell is close to the plasma. As a consequence, present day tokamaks normally need to operate with $q(a)>2$ in order to avoid the $m=2$ kink mode [16]. Note that the external $m=1 \mathrm{kink}$ mode remains out of reach by a safe margin.

Whether a mode is actually unstable depends on the details of the current profile. In order to obtain a stability criterion the integral over the plasma interior in (C.) must be minimized. The minimizing function $\xi_{r}(r)$ is a solution of the Euler equation obtained from (C.),

$$
\frac{d}{d r}\left[\left(\frac{n}{m}-\frac{1}{q}\right)^{2} r^{3} \frac{d \xi_{r}}{d r}\right]=\left(m^{2}-1\right)\left(\frac{n}{m}-\frac{1}{q}\right)^{2} r \xi_{r} .
$$

Note that for $n=0$ this equation is identical to the radial equations for small equilibrium shaping effects, equation (18) in Ref. [14]. Equation (24) is singular in $r=0$ and in $q=m / n$. In the vicinity of the magnetic axis $q(r)$ is approximately constant. Hence, for small $r$ we can find exact solutions to Eq. (24), $\xi_{r}(r) \sim r^{-1 \pm m}$. The solution that is regular at $r=0$ is

$$
\xi_{r} \sim r^{m-1}, \quad \xi_{\theta}=i \xi_{r} .
$$

One sees that for $m=1$ the components $\xi_{r}$ and $\xi_{\theta}$ are constant in the plasma centre, which corresponds to a rigid displacement of the plasma core. For $m>1$, however, the mode amplitude vanishes in the plasma centre. Equation (24), together with (21), gives a good indication of how a global MHD eigenfunction with specific toroidal and poloidal mode numbers looks like in an approximately circular plasma cross section, even if one has to take into account additional effects before the stability criterion for such a mode can be found. An example of this is presented in the next subsection.

\section{The internal $m=1$ kink mode}

We will show in this subsection that for $m=1$ one can also find an unstable mode with $\xi_{r}(a)=0$, i.e., an internal mode. It is clear from the energy functional (22) that the case $m=1$ requires a special treatment. A general calculation shows that the $m=n=1$ mode is in general more unstable than the $m=1$ modes with higher toroidal mode numbers. For simplicity, we will restrict ourselves to the toroidal mode number $n=1$ from the start. The leading order energy functional reduces to

$$
\delta W_{m=1}=\pi^{2} \frac{B_{0}^{2}}{R_{0}} \int_{0}^{a} r^{3}\left|\xi_{r}^{\prime}\right|^{2}\left(1-\frac{1}{q}\right)^{2} d r .
$$

Equation (25) implies that this functional is minimized by $\xi_{r}(r)=$ constant. However, we are looking for an internal mode, $\xi_{r}(a)=0$. Consider therefore a trial function which is constant in the plasma centre, shows a steep step at the radius $r=r_{1}$ where $q=1$, and vanishes for $r>r_{1}$ [17]. Let us call the width of the layer where $\xi_{r}(r)$ changes from its central value to zero $\delta$. We will show now that $\delta W_{m=1}$ vanishes for $\delta \rightarrow 0$. The radial derivative of $\xi_{r}$ is of course large, $\left|\xi_{r}^{\prime}\right|^{2}=\mathcal{O}\left(1 / \delta^{2}\right)$. On the other hand, $q \approx 1$ in this layer, so that $(1-1 / q)^{2}=\mathcal{O}\left(\delta^{2}\right)$. Therefore, small and large factors cancel in the integrand of (26). It follows that $\delta W_{m=1}$ vanishes because the integration interval itself has width $\delta$.

The resulting eigenfunction $\boldsymbol{\xi}(\boldsymbol{x})$ represents a rigid displacement of the plasma inside the $q=1$ surface. It can be understood that the stabilizing magnetic energy terms in $\delta W$ practically vanish by considering the three-dimensional structure of the mode $\boldsymbol{\xi}(\boldsymbol{x})=\boldsymbol{\xi} \exp i(\theta-\phi)$. In the $\phi=0$ plane, the rigid displacement is pointed toward the low field side. At $\phi=\frac{\pi}{2}$ it is directed upward, at $\phi=\pi$ the motion is to the high field side, and at $\phi=\frac{3 \pi}{2}$ it is downward. Summarizing, the motion of the plasma ring with $q<1$ consists of a rigid shift in the horizontal $\phi=0$ direction combined with a tilt with respect to the axis in that direction. In contrast with higher $m, n$ modes, which cause a helical kink deformation, the $m=n=1$ mode does not deform the flux surfaces, and therefore the field line compression and bending terms in $\delta W$ vanish.

The $q \approx 1$ layer, where $\xi_{r}^{\prime}$ is large, requires a more careful discussion, however. Note that the poloidal displace- 
ment, given by Eq. (21), becomes very large for $\delta \rightarrow 0$. This can be visualized as follows. The rigid displacement of the plasma inside the $q=1$ surface pushes it against this surface and the outer plasma, which remains motionless. Since field lines cannot cross each other, and because of incompressibility, the plasma has to flow back at high speed in the thin $q \approx 1$ layer, thus creating extreme convection cells. The plasma motion in the singular layer does not require magnetic energy because the field lines with $q=1$ are closed and have exactly the same helicity as the mode. Hence they can be interchanged freely.

Summarizing, we have minimized the energy functional to the point that $\delta W=\mathcal{O}\left(\varepsilon^{4}\right)$. First, we have chosen $\xi_{\|}$such that there is no plasma compression. Then we have chosen $\xi_{\theta}$ such that there is no field line compression, and finally we have taken $m=n=1$ and we have taken a step-function for $\xi_{r}$ so that there is no field line bending. We have found the general shape of the displacement function, but not yet a stability criterion. In order to find the marginal stability criterion we have to solve the $\mathcal{O}\left(\varepsilon^{4}\right)$ equation $\delta W\left(\boldsymbol{\xi}^{*}, \boldsymbol{\xi}\right)=0$. This requires that the eigenfunction $\boldsymbol{\xi}$ is computed to higher order in $\varepsilon$ than we have done above. In $\mathcal{O}\left(\varepsilon^{4}\right)$, the energy functional contains the following new terms.

1. Terms due to $\nabla p$ and $\boldsymbol{j}_{\perp}$, which are $\mathcal{O}\left(\beta \sim \varepsilon^{2}\right)$ smaller than the terms previously considered, enter the cylindrical model. Hence, the internal kink mode is not a purely current driven mode. In practice, the instability is mainly driven by large pressure gradients in the central plasma (within the $q=1$ surface), while the current is somewhat stabilizing if it is peaked in the centre.

2. The toroidal curvature of the plasma has to be taken into account. This leads to corrections to the cylindrical approximation. An example of such a correction is the Shafranovshift, which is of the order $\Delta(r)=\mathcal{O}(\varepsilon r)$ in the low- $\beta$ ordering. This shift contains "toroidal" terms due to the pressure and the current.

3. Another consequence of the toroidal shape is that eigenmodes are not exact poloidal harmonics. In the case of the internal $m=1 \mathrm{kink}$ instability, the mode is dominantly the $m=1$ harmonic, as discussed in the cylindrical approximation. In addition, however, there are small sidebands. They have poloidal mode numbers $m=0$ and $m=2$, and are $\mathcal{O}(\varepsilon)$ smaller than the $m=1$ harmonic of the instability. These two sideband harmonics as well avoid plasma compression and field line compression. In suitable coordinates they obey the cylindrical equation (24) with $m=0$ and $m=2$, respectively. They cannot avoid field line bending however (which only the $m=1$ step-function can do). Hence these small harmonics contribute to $\mathcal{O}\left(\varepsilon^{4}\right)$ to the energy, i.e., they are just as important for the stability criterion as the main $m=1$ harmonic.

4. In subsection A. we concluded that the field compression energy was minimized to $\mathcal{O}\left(\varepsilon^{4}\right)$. Therefore we must presently take into account that the magnetic field is not entirely incompressible.
The derivation of the final stability criterion requires a lot of algebra and yields a rather complicated equation. The current profile enters in a non-trivial way, for instance, because the general solution of Eq. (24) for the $m=2$ harmonic is not a closed form. The pressure plays a more straightforward role. The final form of $\delta W$ is a quadratic function of

$$
\beta_{p}\left(r_{1}\right) \equiv-2 \frac{R_{0}^{2} q^{2}}{B_{0}^{2} r_{1}^{4}} \int_{0}^{r_{1}} p^{\prime} r^{2} d r
$$

This quantity represents the total available kinetic energy within $r=r_{1}$. A simple form for $\delta W$ can be obtained if we consider a parabolic current profile $j_{\phi}(r)$, and if we assume that $q(r)$ in the centre does not differ very much from unity,

$$
|1-q(0)| \ll 1, \quad q(0)<1 .
$$

Then, the $m=1$ internal kink mode is mainly pressure driven and the potential energy is approximately [18]

$$
\delta W \approx 6 \pi^{2} \frac{B_{0}^{2} r_{1}^{4}}{R_{0}^{3}}\left|\xi_{r}(0)\right|^{2}[1-q(0)]\left[\beta_{\text {crit }}^{2}-\beta_{p}^{2}\left(r_{1}\right)\right]
$$

where $\beta_{\text {crit }}^{2}=\frac{13}{144}$. One sees that instability, $\delta W<0$, occurs if the driving force $\beta_{p}$ exceeds the threshold value $\beta_{\text {crit }} \approx$ 0.3 .

Equation (27) qualitatively matches the observations of sawtooth oscillations in tokamaks. Sawteeth generally occur when the central plasma temperature rises due to auxiliary plasma heating. When the central temperature reaches a certain level, the plasma core becomes unstable and quickly, typically within $100 \mu \mathrm{s}$ in the hottest tokamak plasmas, looses all its excess energy. The temperature profile after the crash is usually completely flat in the central plasma, or even hollow. It has been observed that the instability initially is a fast $m=1, n=1$ displacement of the hot plasma core, and that the part of the plasma where this motion takes place and where eventually the temperature is reduced indeed matches the $q<1$ region.

The application of the internal $m=1$ kink model to sawtooth collapses has many limitations, though. Firstly, we have seen that the motion at the $q=1$ surface is highly singular. The motion minimizes $\delta W$, but in the case of an unstable mode the fast motion near $r=r_{1}$ corresponds to a large kinetic energy, and hence the actual growth rate of the mode is limited. However, other processes can take place. The singular behaviour was due to the fact that flux surfaces with $q<1$ were pushed against flux surfaces with $q>1$. In ideal MHD this process cannot proceed, and the ideal $m=1$ mode is expected to saturate nonlinearly at a very small amplitude. It is here that resistivity, however small in a hot plasma, becomes important [19]. In the singular layer it can cause pairs of approaching flux surfaces with $q<1$ and $q>1$ to coalesce and to form a topologically distinct magnetic island [20]. In the process the different temperatures of the two merging surfaces are of course averaged. In 
Kadomtsev's model of the sawtooth relaxation [21], the reconnection process proceeds until the entire plasma core is replaced by the island and and as a result $q=1$ in the entire central region. Also the resulting temperature and density profiles are practically flat in this region. However, this model does not match all observations of sawteeth. For instance, sawtooth crash times much shorter than the prediction of the Kadomtsev model have been observed. In addition, measurements of the central $q$-profile have been made which indicate that sawteeth can occur with $q(0)<0.8$ throughout the sawtooth cycle [22]. Ref. [28] gives a review of $m=1$ mode theory.

\section{PRESSURE DRIVEN INSTABILITIES}

\section{A. Introduction}

In this section modes are considered that are mainly driven by the pressure gradient, i.e., modes for which the fifth term in the intuitive from of the energy functional (19) is the dominant destabilizing contribution. One sees that this term can be destabilizing when $\boldsymbol{\xi} \cdot \nabla p$ and $\boldsymbol{\xi} \cdot \boldsymbol{\kappa}$ have the same sign, and this effect is strongest when the vectors $\nabla p$ and $\kappa$ are in the same direction (unfavourable curvature). To clarify this situation for tokamak equilibria, we rewrite the momentum balance equation $\nabla p=\boldsymbol{j} \times \boldsymbol{B}$ as

$$
\nabla p=B^{2} \boldsymbol{\kappa}-\frac{1}{2} \nabla_{\perp} B^{2}, \quad\left(\nabla_{\perp}=\nabla-\boldsymbol{b} \boldsymbol{b} \cdot \nabla\right)
$$

Suppose for a start that the plasma pressure is balanced by the magnetic pressure, the second term on the right. However, due to the toroidal geometry $B$ has an overall $1 / R$ dependence. Therefore, $\nabla \frac{1}{2} B^{2}$ is necessarily directed outward at the low field side of the torus, i.e., magnetic pressure cannot prevent a plasma column from expanding in the $\nabla R$ direction. Hence, at the low field side the magnetic curvature term (the pull of the field lines) must balance the pressure gradient. It is clear from Eq. (19) that this results in a region of bad curvature $(\boldsymbol{\kappa} \cdot \nabla p>0)$.

In such a region a flux tube is pulled inward by its own tension and pushed outward by the pressure gradient. Thus, if a flux tube of higher pressure could interchange position with a flux tube of lower pressure, their changes of the magnetic energy would cancel while pressure energy would be released. If the motions of the flux tubes in a bad curvature region were not constrained in other ways, instabilities could occur on an arbitrarily small scale. The process would be analogous to the Rayleigh-Taylor instability.

Two effects prevent this process from happening universally. Firstly, field lines in a tokamak pass regions of favourable curvature (where the magnetic pressure confines the plasma) as well as bad curvature regions. Indeed, the bad curvature region in a tokamak plasma can be minimized by a suitable combination of outward pointing triangularity and vertical elongation. Secondly, in the presence of magnetic shear, field lines on neighbouring flux surfaces are not perfectly aligned so that interchange of field lines requires some magnetic energy.

\section{B. Ballooning stability}

The pressure-driven modes that are generally the most unstable (and which give rise to the most stringent stability limits) are the so-called ballooning modes. These modes owe their name to their tendency to have a larger amplitude in parts of the plasma where the destabilization originates, in a tokamak usually the low field side. Such a spatial variation of the amplitude needs to be of a very particular nature in order to avoid the strong stabilizing effect of the magnetic field. In contrast, kink modes avoid this stabilizing effect by having an almost constant amplitude on a flux surface. Another contrast is that for ballooning modes, higher toroidal mode numbers $n$ are more unstable. Ballooning modes have a rather complicated spatial structure because their stability depends on geometric details of the plasma, especially the curvature of the field lines. We shall avoid this complexity by focussing on the stability limit (usually a limit on the pressure gradient). Exactly at the stability boundary, which is found in the limit $n \rightarrow \infty$. The corresponding modes are highly localized in radius, so that we obtain stability criteria for each flux surface separately.

We shall find the marginally stable modes by mimimizing $\delta W(\boldsymbol{\xi})$, which is equivalent to solving Eq. (18). In terms of the minimized value of the energy functional, the criterion for instability is then $\delta W_{\min }<0$. Our approach will be to consider only a specific class of instabilities, with high poloidal and toroidal mode numbers $m$ and $n$. As we will see, such modes tend to be rather localized in $r$. We are in effect considering the situation where a gradually evolving (intensively heated) plasma equilibrium, when it reaches stability limits, initially does so in a small volume. Therfore, we expect the first instability to occur to be a localized one. Though this is the most straightforward situation, we will see in the next section that some of the most common instabilities affect a rather large plasma volume, what makes them more dangerous for confinement.

Here, we will consider instabilities in the limit $n \rightarrow$ $\infty$. Connor, Hastie, and Taylor [23] have treated this limit, neglecting $\mathcal{O}\left(1 / n^{2}\right)$ but retaining $\mathcal{O}(1 / n)$ terms. They have shown that the $\mathcal{O}(1 / n)$ terms are always stabilizing. In this sense, the most unstable modes are the ones with $n=\infty$. Therefore, in this section we will take the limit $n \rightarrow \infty$ and neglect the $\mathcal{O}(1 / n)$ terms right away. The minimization of $\delta W$ proceeds in five steps [7], bringing us from the threedimensional vector equation $\boldsymbol{F}(\boldsymbol{\xi})=0$ to a one-dimensional equation for one scalar function.

1. The plasma compression term in (19) vanishes by minimization with respect to $\xi_{\|}$, which is chosen in such a way that $\nabla \cdot \boldsymbol{\xi}=0$ almost everywhere in the plasma.

2. One cannot take the limit $n \rightarrow \infty$ straight away. If one considers high mode numbers $m$ and $n$, one quickly sees that the stabilizing contribution $\left|\boldsymbol{B} \cdot \nabla \boldsymbol{\xi}_{\perp}\right|^{2}$ to the field line bend- 
ing energy $\frac{1}{2} \int d^{3} x\left|\boldsymbol{Q}_{\perp}\right|^{2}$ is proportional to $(n-m / q)^{2}$ and is therefore much larger than the destabilizing terms in (19). Hence, the most unstable modes will tend to be aligned to the field lines, $m \approx n q$. In more general words, $\boldsymbol{\xi}_{\perp}$ will vary strongly only perpendicular to the field lines $\left(\boldsymbol{k}_{\perp} \gg 1 / a\right)$ while varying slowly, on the scale of the machine size, along the field lines $\left(k_{\|} \sim 1 / a\right)$. We implement this ordering by means of an eikonal representation for $\boldsymbol{\xi}_{\perp}[23,24]$,

$$
\boldsymbol{\xi}_{\perp}(\psi, \theta, \phi)=\boldsymbol{\xi}_{\perp}(\psi, \theta) e^{i n S}, \quad \boldsymbol{B} \cdot \nabla S(\psi, \theta, \phi)=0 .
$$

Since $n$ is large, $e^{i n S}$ is a rapidly varying function, while $\boldsymbol{\xi}_{\perp}(\psi, \theta)$ varies slowly.

$$
|n \nabla S| \gg \frac{1}{a}, \quad\left|\nabla \boldsymbol{\xi}_{\perp}\right| \sim \frac{\left|\boldsymbol{\xi}_{\perp}\right|}{a} .
$$

This formalism serves its purpose. The large quantity $\nabla S$ does not enter the field line bending energy as one can see by working out $\boldsymbol{Q}$ in terms of $\boldsymbol{\xi} e^{i n S}$,

$$
\boldsymbol{Q}_{\perp}=e^{i n S}\left[\nabla \times\left(\boldsymbol{\xi}_{\perp} \times \boldsymbol{B}\right)\right]_{\perp} .
$$

The energy functional becomes

$$
\begin{aligned}
\delta W=\frac{1}{2} \int d^{3} x[ & \left|\boldsymbol{Q}_{\perp}\right|^{2}+B^{2}\left|i n \nabla S \cdot \boldsymbol{\xi}_{\perp}+\nabla \cdot \boldsymbol{\xi}_{\perp}+2 \boldsymbol{\kappa} \cdot \boldsymbol{\xi}_{\perp}\right|^{2} \\
& -2\left(\boldsymbol{\xi}_{\perp} \cdot \nabla p\right)\left(\boldsymbol{\xi}_{\perp}^{*} \cdot \boldsymbol{\kappa}\right) \\
& \left.-j_{\|} \boldsymbol{\xi}_{\perp}^{*} \times \boldsymbol{b} \cdot \boldsymbol{Q}_{\perp} e^{-i n S}\right]
\end{aligned}
$$

3. At this stage the mode number $n$ still appears in the field compression (second term) and leads to the large stabilizing energy contribution $\frac{1}{2} n^{2} \int d^{3} x B^{2}\left|\nabla S \boldsymbol{\xi}_{\perp}\right|^{2}$. In order to keep this term finite, the perturbation $\xi_{\perp}$ must have the general form

$$
\boldsymbol{\xi}_{\perp}=\boldsymbol{\xi}_{\perp 0}+\frac{\boldsymbol{\xi}_{\perp 1}}{n}, \quad \boldsymbol{\xi}_{\perp 0} \equiv \frac{X}{B} \boldsymbol{b} \times \nabla S
$$

where $X(\psi, \theta)$ is a new scalar function independent of $\boldsymbol{\xi}_{\perp 1}$. One may verify that with $\xi_{\perp}$ of the form (29), the term of concern in Eq. (28) is indeed finite,

$$
i n \nabla S \cdot \boldsymbol{\xi}_{\perp}=i \nabla S \cdot \boldsymbol{\xi}_{\perp 1} .
$$

4. Now we can take the limit $n \rightarrow \infty$. In this limit

$$
\begin{aligned}
\boldsymbol{Q}_{\perp} & =\nabla \times[(X \boldsymbol{b} \times \nabla S) \times \boldsymbol{b}]_{\perp} \\
& =\nabla \times(X \nabla S)_{\perp} \\
& =\nabla \times(X \nabla S)-\boldsymbol{b} \boldsymbol{b} \cdot \nabla \times(X \nabla S) \\
& =(\boldsymbol{b} \cdot \nabla X) \boldsymbol{b} \times \nabla S .
\end{aligned}
$$

The $\boldsymbol{j}_{\|}$-term vanishes because

$$
\left(\boldsymbol{\xi}_{\perp 0}^{*} \times \boldsymbol{b}\right) \cdot \boldsymbol{Q}_{\perp}=\frac{X^{*}}{B} \nabla S \cdot(\boldsymbol{b} \cdot \nabla X) \boldsymbol{b} \times \nabla S=0 .
$$

Thus the energy becomes

$$
\begin{aligned}
\delta W=\frac{1}{2} \int d^{3} x[ & |\nabla S|^{2}|\boldsymbol{b} \cdot \nabla X|^{2} \\
& +B^{2}\left|i \boldsymbol{\xi}_{\perp 1} \cdot \nabla S+\nabla \cdot \boldsymbol{\xi}_{\perp 0}+2 \boldsymbol{\kappa} \cdot \boldsymbol{\xi}_{\perp 0}\right|^{2} \\
& \left.-2(\boldsymbol{B} \times \nabla S \cdot \nabla p)(\boldsymbol{B} \times \nabla S \cdot \boldsymbol{\kappa})|X|^{2}\right] .
\end{aligned}
$$

The variable $\boldsymbol{\xi}_{\perp 1}$ appears only in the second term. Therefore, the energy can be minimized with respect to $\boldsymbol{\xi}_{\perp 1}$ simply by choosing $i \boldsymbol{\xi}_{\perp 1} \cdot \nabla S=-\nabla \cdot \boldsymbol{\xi}_{\perp 0}-2 \boldsymbol{\kappa} \cdot \boldsymbol{\xi}_{\perp 0}$, thus eliminating the field compression term altogether.

5. The remaining functional contains only one variable $X$ and its derivative along the field lines, $\boldsymbol{b} \cdot \nabla X$. It is essentially one-dimensional since it does not contain the radial derivative of $X$. We can therefore consider a potential energy functional on each flux surface separately

$$
\begin{aligned}
\delta W=\int d \theta J[ & |\nabla S|^{2}|\boldsymbol{b} \cdot \nabla X|^{2}- \\
& \left.2(\boldsymbol{B} \times \nabla S \cdot \nabla p)(\boldsymbol{B} \times \nabla S \cdot \boldsymbol{\kappa})|X|^{2}\right]
\end{aligned}
$$

where the Jacobian is $J(\psi, \theta, \phi)=(d \ell / d \theta) / B_{p}$. The derivative in the magnetic field direction can be rewritten as $\boldsymbol{b} \cdot \nabla X=(\partial X / \partial \theta) / J B$. Hence the Euler equation for $X$, associated with the functional (30) is given by

$\frac{1}{J} \frac{\partial}{\partial \theta}\left(\frac{|\nabla S|^{2}}{J B^{2}} \frac{\partial X}{\partial \theta}\right)+2(\boldsymbol{B} \times \nabla S \cdot \nabla p)(\boldsymbol{B} \times \nabla S \cdot \boldsymbol{\kappa}) X=0$.

It is a linear second order differential equation in the poloidal coordinate $\theta$. Note that the combination $J^{-1} \partial / \partial \theta$ is independent of the definition of the poloidal coordinate.

How have we lost the radial dimension of our stability problem? It is not a straightforward matter of invariance, as is the case with the $\phi$ coordinate. The modes we consider have $\boldsymbol{b} \cdot \nabla=k_{\|} \ll\left|\boldsymbol{k}_{\perp}\right|$, which is a non-trivial situation in a tokamak because of the presence of magnetic shear: $q$ varies with the radius. The answer is that we have hidden these difficulties in our assumption that we can find an eikonal function that satisfies $\boldsymbol{B} \cdot \nabla S=0$. In the orthogonal flux coordinates this condition can be written as

$$
\frac{F}{R^{2}} \frac{\partial S}{\partial \phi}+\frac{1}{J} \frac{\partial S}{\partial \theta}=0
$$

We now consider a single toroidal harmonic, $S(\psi, \theta, \phi)=$ $-\phi+S(\psi, \theta)$, and obtain solutions by integrating Eq. (C.) with respect to $\theta$,

$$
S=-\phi+F \int_{\theta_{0}}^{\theta} \frac{J}{R^{2}} d \theta .
$$

The integration boundary $\theta_{0}$ is a free parameter in our solution. The energy functional should be minimized with respect to $\theta_{0}$ as well in order to find the most unstable mode. For up-down symmetric equilibria the minimizing 
value often is $\theta_{0}=0$. We recognize in (33) an incomplete version of the integral that yields the safety factor, $q(\psi)=(F / 2 \pi) \oint d \theta J / R^{2}$. It is clear now that the function $S$ satisfies

$$
S(\psi, \theta+2 \pi, \phi)=S(\psi, \theta, \phi)+2 \pi q .
$$

Let us consider the value of $S$ on two neighbouring field lines with a small spatial separation. In the presence of magnetic shear these lines will differ slightly, by $\Delta q$. Then, since $n$ is large, the values of $e^{i n S}$ on these field lines will diverge strongly from each other, at the rate $n \Delta q$, when $\theta$ increases. Therefore $e^{i n S}$ depends so strongly on the radial coordinate that almost all radial dependence of the vector $\xi$ is contained in the factor $e^{i n S}$.

It seems that we have treated complicated behaviour as a function of the radius in a compact way. There are, however, two problems with this approach. The most obvious problem is that the radial dependence of $e^{i n S}$ is strongly oscillatory and does not give rise to a radially localized eigenfunction $\boldsymbol{\xi}$, although Eq. (31) applies to any individual flux surface. The second problem arises because for irrational values of $q$, the function $e^{i n S}$ is not periodic in $\theta$, and neither is $\nabla S$. Hence, the constraint (32) is incompatible with periodicity. Even if a periodic solution $X(\theta)$ can be found, the associated displacement vector $\boldsymbol{\xi}(\psi, \theta, \phi)$ is not periodic in $\theta$ and hence not acceptable as a physical perturbation of the equilibrium. We will see that the two problems are related and that the solution of the second problem takes care of the first one as well.

A practical solution of these difficulties has been given by Connor, Hastie, and Taylor [24]. The basic idea is to give up the periodicity in $\theta$ in the energy functional (30), and allow the generalized angle $\theta$ run over the entire real axis $(-\infty, \infty)$. Also the Euler equation (31) should be considered as a differential equation over the real axis, with its boundary conditions in $\theta= \pm \infty$. With this Ansatz, we make four observations.

1. The linear second order equation (31) has in general two independent solutions, one of which vanishes for $\theta \rightarrow-\infty$, the other for $\theta \rightarrow \infty$. Marginal stability corresponds to special values of the equilibrium quantities for which the equation has a solution that vanishes for $\theta \rightarrow \pm \infty$ simultaneously. This we require since the eigenmode must have a finite energy content. Of course, away from marginal stability one still requires that the eigenfunction is finite, and a kinetic energy term with the proper value of $\omega^{2}$ has to be added to Eq. (31) to make this possible.

2. Now consider the eigenfunction $\boldsymbol{\xi}_{Q}(\boldsymbol{x})$ associated with a finite solution $X(\theta)$ of Eq. (31). This eigenfunction is not periodic in $\theta$ and hence not physical but it does satisfy the equation of motion at marginal stability (18), $\boldsymbol{F}\left(\boldsymbol{\xi}_{Q}\right)=0$, everywhere in the extended $(\psi, \theta, \phi)$ space. The function $\boldsymbol{\xi}_{Q}$ is called a quasi-mode. Note that the force operator itself is periodic, $\boldsymbol{F}(\psi, \theta, \phi)=\boldsymbol{F}(\psi, \theta+2 \pi, \phi)$. Hence, if the shifted quasimode $\boldsymbol{\xi}_{Q}(\psi, \theta+2 \pi k, \phi)$ is a solution of the force balance equation as well.
3. The force operator $\boldsymbol{F}$ is a linear operator.

4. The infinite sum

$$
\boldsymbol{\xi}(\psi, \theta, \phi)=\sum_{k=-\infty}^{\infty} \boldsymbol{\xi}_{Q}(\theta+2 \pi k)
$$

is clearly periodic in $\theta$ if it converges. We have effectively wound the infinite $\theta$-axis around the unit circle, summing the contributions to the eigenfunction on it in the process. It is clear that the proper boundary conditions for the generalized Euler equation have to include $X \rightarrow 0$ for $\theta \rightarrow \pm \infty$ in order to have a convergent sum.

We will now combine these four observations. According to observation 2, all terms in the sum (34) satisfy the equation of motion at marginal stability and point 3 (linearity) guarantees that the sum (34), if it exists, also satisfies this equation. Observation 1 states that the necessary boundary conditions for the existence of the sum, $X(\theta \rightarrow \pm \infty)=0$, are satisfied in the case of marginal stability. Finally, according to point 4 , expression (34) defines a periodic solution of the marginal stability equation (18).

Expression (34) is a sum over many terms that have different values of the non-periodic function $e^{i n S}$, which contains the radial dependence of $\boldsymbol{\xi}$. Note that the individual quasimodes are not bounded in the radial direction since the exponent vanishes nowhere. Fortunately, as pointed out by Pegoraro and Schep [25], the sum (34) is radially localized at the flux surface where we have solved Eq. (31). They show that one can make a Fourier transformation from an extended poloidal coordinate to an extended radial coordinate, and hence the poloidal and radial widths of the mode are inversely proportional. Loosely speaking, ballooning modes tend to be rather localized in the radial direction when they have an extended range in the poloidal coordinate.

Satisfied with the above picture, one can find the stability at a given flux surface by solving Eq. (31) for that particular value of $\psi$. The coefficients in the differential equation can be computed numerically from the $q$-profile, the pressure gradient, and the shape of the flux surface. One usually obtains such data from a numerical equilibrium solver (solver of the Grad-Shafranov equation) though in principle one does not need a solution in the entire plasma in order to find the stability of a single flux surface.

\section{The Mercier criterion and $\beta$-limits}

Straightforward integration of the ballooning equation (31) is often numerically demanding because the coefficients contain "slowly" and "quickly" varying functions of $\theta$. The quantity $|\nabla S|$ is a steadily increasing function for $\theta \rightarrow \infty$. On the other hand, quantities $\kappa, J$, and $B$ are periodic functions of $\theta$. They can be considered rapidly oscillating functions of $\theta$ on the scale on which $|\nabla S|$ becomes large. One can find the asymptotic behaviour of solutions $X(\theta)$ by a procedure of averaging over the oscillating terms. For large 
$\theta$ equation (31) takes the form

$$
\frac{d}{d \theta}\left(\theta^{2} \frac{d X}{d \theta}\right)+D_{M} X=0
$$

where the so-called Mercier constant $D_{M}$ is a complicated function of the equilibrium quantities on the flux surface. It follows that the general asymptotic form of $X(\theta)$, i.e., the general solution of (35) is

$$
X=c_{+} \theta^{\lambda_{+}}+c_{-} \theta^{\lambda_{-}}, \quad \lambda_{ \pm} \equiv-\frac{1}{2} \pm \sqrt{\frac{1}{4}-D_{M}} .
$$

If $D_{M}<\frac{1}{4}$ there is a large solution $\sim \theta^{\lambda_{+}}$and a small solution $\sim \theta^{\lambda_{-}}$. The large solution makes the integral (28) infinite, so only the small solution is allowed. The ballooning criterion is precisely the condition under which one solution of (31) is asymptotically small, $\sim \theta^{\lambda_{-}}$, for both $\theta \rightarrow-\infty$ and $\theta \rightarrow \infty$. The situation is different if $D_{M}>\frac{1}{4}$. In this case both exponents $\lambda_{ \pm}$are complex and lead to oscillatory solutions with a finite energy content. In this case all solutions of Eq. (31) are allowed, i.e., there is always instability and there is no ballooning stability boundary. It can be shown that this situation is unstable by constructing radially localized trial functions $\boldsymbol{\xi}_{T}$ for which $\delta W\left(\boldsymbol{\xi}_{T}^{*}, \boldsymbol{\xi}_{T}\right)<0$, instead of constructing proper eigenfunctions as required for the ballooning stability criterion. The condition

$$
D_{M}<\frac{1}{4} \text {. }
$$

is the Mercier criterion [26]. It is a necessary condition for stability. If it is violated instability occurs. If it is satisfied, the plasma can still be unstable to ballooning modes. A simple closed form for $D_{M}$ can be derived for large aspect-ratio circular flux surfaces. In this approximation the ballooning equation takes the form

$$
\frac{d}{d \theta}\left(\left(1+P^{2}\right) \frac{d X}{d \theta}\right)+\alpha(\cos \theta+P \sin \theta+V) X=0,
$$

where the "shear integral" $P$ originates from $|\nabla S|$ in Eq. (31) and the potential term $V$ gives the effect of the "magnetic well" in the plasma centre. They are given by

$$
P=s \theta-\alpha \sin \theta, \quad V=\varepsilon\left(1-\frac{1}{q^{2}}\right) .
$$

The quantities

$$
s=\frac{r}{q} \frac{d q}{d r}, \quad \alpha=-2 \frac{R q^{2}}{B^{2}} \frac{d p}{d r},
$$

are the magnetic shear and the normalized pressure gradient. In this model the Mercier index is

$$
D_{M}=\varepsilon \frac{\alpha}{s^{2}}\left(\frac{1}{q^{2}}-1\right) .
$$

Combining Eqs. (36) and (39) one sees that Mercier instability requires $q$ below unity, low shear, and a large pressure gradient.
In the case that the Mercier criterion indicates stability, $D_{M}<\frac{1}{4}$, one can test Eq. (37) for ballooning instability. Also in the case of ballooning modes one finds that generally low shear is more unstable than high shear. This is understandable since instabilities tend to align with the magnetic field on a given flux surface. The rate at which the mode and the magnetic field become misaligned on neighbouring flux surfaces is proportional to the magnetic shear. The pressure gradient is destabilizing, and in general large $\alpha$ will lead to instability. Surprisingly, Eq. (37) also implies that for very large values of $\alpha$ and not too high shear a second regime of stability exists. For such high pressure the Shafranov shift gives rise to a very asymmetric plasma cross section, with flux surfaces tightly pushed together a the low field side. In that region the local magnetic shear (not the flux quantity $s$ defined in (38)) becomes high and stabilizes ballooning modes. It turns that if the plasma cross section is strongly triangular, the second stability regime is also accessible for lower values of the pressure gradient, provided the magnetic shear is negative.

Perhaps the most powerful application of ballooning stability analysis is the numerical optimization of the total plasma $\beta$ by varying the $p$ and $q$ profiles and the plasma shape, under the constraint that all flux surfaces be Mercier and ballooning stable. The best known of such studies has been carried out by Troyon et al. [27]. They have varied the $p$ and $q$ profiles as well as the plasma elongation and triangularity. Their stability criteria involve ballooning stability on every flux surface. In addition, there are the constraints $q(a)>2$ in order to prevent $m=2$ external kink modes and $q(0)>1$ in order to satisfy the Mercier criterion and to avoid $m=1$ internal kink modes. The resulting $\beta$-limit is

$$
\beta=0.028 \frac{I_{0}}{a B_{0}},
$$

where the units are $I_{0}(\mathrm{MA}), a(\mathrm{~m})$, and $B_{0}(\mathrm{~T})$. This result is called the Troyon limit. It can be of limited validity for instance when instabilities that are not listed above play a role. A positive aspect is that a tokamak discharge may exceed the Troyon limit if part of the plasma is in the second stability regime.

In experiments, ballooning limits are often "soft". This means that when attempts are made to increase the tokamak plasma pressure with intense heating, a certain pressure gradient cannot be exceeded. This is probably related to the fact that the instabilities near the ballooning stability limit are very localized. The small amplitudes of the instabilities are then seen as enhanced heat loss, not as a disruptive instability. However, ballooning modes have also been associated with some of the Edge Localized Modes (ELMs) observed in tokamaks. These modes appear repetitive as a sudden loss of the outer layer of plasma from the tokamak. 


\section{ACKNOWLEDGMENT}

This work, supported by the European Communities under the contract of Association between EURATOM/FOM, was carried out within the framework of the European Fusion Programme with financial support from NWO. The views and opinions expressed herein do not necessarily reflect those of the European Commission.

\section{REFERENCES}

1. S. Sharapov "MHD and fast particle effects", these proceedings.

2. H. Wilson "Neo-classical tearing mode", these proceedings.

3. R.M. Kulsrud, "General stability theory in plasma physics," in "Proc. of the Int. School of Physics "Enrico Fermi", Course XXV, "Advanced Plasma Theory", (edited by M.N. Rosenbluth), Academic Press, New York, (1964).

4. R.M. Kulsrud, "MHD description of plasma," in "Handbook of Plasma Physics" (edited by M.N. Rosenbluth and R.Z. Sagdeev), North-Holland, Amsterdam, Vol. 1, p. 115, (1983).

5. I.B. Bernstein, E.A. Frieman, M.D. Kruskal, and R.M. Kulsrud, "An energy principle for hydromagnetic stability problems," Proc. Roy. Soc. A 244, 17, reprinted in "Magnetohydrodynamic Stability and Thermonuclear Confinement" (edited by A (1958). Jeffrey and T. Taniuti), Academic Press, New York (1966).

6. J.P. Freidberg, "Ideal magnetohydrodynamic theory of magnetic fusion systems," Rev. Mod. Phys. 54, 801 (1982).

7. J.P. Freidberg, "Ideal Magnetohydrodynamics", Plenum Press, New York (1987).

8. J.P. Goedbloed and S. Poedts, "Principles of Magnetohydrodynamics", Cambridge University Press (2004).

9. I.B. Bernstein, "The variational principle for problems of ideal magnetohydrodynamic stability," in "Handbook of Plasma Physics" (edited by M.N. Rosenbluth and R.Z. Sagdeev), North-Holland, Amsterdam, Vol. 1, p. 421, (1983).

10. K. Hain, R. Lüst, and A. Schlüter, "Zur Stabilität eines Plasmas," Z. Naturforschung. 12, 833 (1957).

11. G. Laval, C. Mercier, and R. Pellat, "Necessity of the energy principles for magnetostatic stability," Nucl. Fusion 5, 156 (1965).

12. W.A. Newcomb, "Hydromagnetic stability of a diffuse linear pinch," Ann. Phys. (NY) 10, 232 (1960).

13. V.D. Shafranov, "Hydromagnetic stability of a current carrying pinch in a Strong Longitudinal Field," Zhurnal Tekhnicheskoi Fiziki 40, 241 (1970) [Sov. Phys.Techn. Phys. 15, 175 (1970)].

14. H.J. de Blank, "Plasma equilibrium in tokamaks," and references therein, these proceedings.
15. J.A. Wesson, "Hydromagnetic stability of tokamaks," Nucl. Fusion 18, 87 (1978).

16. H.R. Koslowski, "Operational limits and limiting instabilities in tokamak machines," these proceedings.

17. M.N. Rosenbluth, R.Y. Dagazian, and P.H. Rutherford, "Non-linear properties of the internal kink instability in the cylindrical tokamak," Phys. Fluids 16, 1894 (1973).

18. M.N. Bussac, R. Pellat, D. Edery, and J.L. Soule, "Internal kink modes in toroidal plasma with circular cross-section," Phys. Rev. Lett. 35, 1638 (1975).

19. H.P. Furth, J. Killeen, and M.N. Rosenbluth, "Finite resistivity instabilities of a sheet pinch," Phys. Fluids 6, 459 (1963).

20. R.B. White, "Resistive instabilities and field line reconnection," in "Handbook of Plasma Physics" (edited by M.N. Rosenbluth and R.Z. Sagdeev), North-Holland, Amsterdam, Vol. 1, p. 611, (1983).

21. B.B. Kadomtsev, "Disruptive instability in tokamaks," Fizika Plasmy 1 (1975) 710 [Sov. J. Plasma Phys. 1 (1976) 389].

22. H. Soltwisch et al., in Plasma Physics and Controlled Nuclear Fusion Research 1986, Proceedings of the 11th International Conference, Kyoto (IAEA, Vienna, 1987), Vol. 1, p. 263.

23. J.W. Connor, R.J. Hastie, and J.B. Taylor, "High mode number stability of axisymmetric toroidal plasmas," Proc. Roy. Soc. A 365, 1 (1979).

24. J.W. Connor, R.J. Hastie, and J.B. Taylor, "Shear, periodicity, and plasma ballooning modes," Phys. Rev. Lett. 40, 396 (1978).

25. F. Pegoraro and T.J. Schep, "Low-frequency modes with high toroidal mode numbers: A general formulation," Phys. Fluids 24, 478 (1981).

26. C. Mercier, "Un critère nécessaire de stabilité hydromagnetique pour un plasma en symétrie de révolution," Nucl. Fusion 1, 47 (1960).

27. F. Troyon, R. Gruber, H. Sauremann, S. Semenzato, and S. Succi, "MHD limits to plasma confinement," Plasma Phys. Controlled Fusion 26, 209 (1984).

28. S. Migliuolo, "Theory of ideal and resistive $\mathrm{m}=1$ modes in tokamaks," Nucl. Fusion 33, 1721 (1993). 